\title{
Iron, Copper, Zinc, Magnesium, and Calcium in Postmortem Brain Tissue from Schizophrenic Patients
}

\author{
Johannes Kornhuber, Klaus W. Lange, Paul Kruzik, Wolf-Dieter Rausch, \\ Eberhard Gabriel, Kurt Jellinger, and Peter Riederer
}

The regional distribution of iron, copper, zinc, magnesium, and calcium in postmortem brain of schizophrenic patients was compared with that of matched controls. In none of the brain regions investigated (caudate nucleus, hippocampus, amygdala, cortex, corpus mamillare, gyrus cinguli, and hypothalamus) were significant differences observed between these two groups. In the total group, region-specific differences were found for iron, copper, zinc, and calcium, but not for magnesium. Gender differences were observed only for zinc. There was no correlation between a neuroleptic-free period before death and the content of any of the metals investigated, except for a positive correlation between copper in the hippocampus and a neuroleptic-free period. The results of the present study suggest that there are no profound differences in the content of iron, copper, zinc, magnesium, and calcium in postmortem brains between controls and schizophrenic patients.

Key Words: Iron, copper, zinc, magnesium, calcium, schizophrenia, human brain, glutamate hypothesis, tardive dyskinesia, neuroleptic medication

\section{Introduction}

Transition metals such as iron, copper, and zinc are known to play a crucial role in various physiological functions in mammalian brains, for example, in prosthetic groups of metalloenzymes and in the control of gene expression (O'Halloran 1993). Transition metals have been linked primarily to neurological disorders such as Wilson's disease, Hallervorden-Spatz syndrome, Parkinson's disease and

From the Department of Psychiatry, University of Würzburg, Germany (IK, KWL, PR); the Institute of Medical Chemistry, Veterinary Medical University (PK, W-DR); the Psychiatric Hospital-Baumgarner Höhe, (EG); and the Ludwig Boltzmann Institute for Clinical Neurobiology. Vienna, Austria (KJ).

Address reprint requests to Johannes Kornhuber, MD, Nervenklinik, Universität Würzburg, Füchsleinstraße 15, D-97080 Würzburg, Germany.

Received Sepiember 27, 1993; revised January 3, 1994.
Alzheimer's disease (e.g., Riederer et al 1989; Constantinidis 1991; Good et al 1992). Cr.sidering the critical role of transition metals in enzyme and receptor function, however, excess or deficiency of these elements might also be related to the pathophysiology of schizophrenia.

Zinc is unevenly distributed within the brain with particularly high concentrations in the hippocampal mossy fiber system, where it acts as a neuromodulator (Xie and Smart 1991). Interactions with inhibitory (Smart and Constanti 1990) and excitatory amino acid neurotransmission (Yet et al 1990) are well known. Although low to moderate concentrations of zinc attenuate $\boldsymbol{N}$-methyl-D-aspartate (NMDA)mediated and enhance quisqualate-mediated neurotoxicity (Koh and Choi 1988), high concentrations of zinc are neurotoxic (Yokoyama et al 1986). Zinc deficiency has been hypothesized to be an important factor in the pathogenesis 
of schizophrenia (Kimura and Kumura 1965; Pfeiffer and lliev 1972; Andrews 1990).

Enhanced concentrations of zinc could be linked to the pathophysiology of schizophrenia, too. The glutamate hypothesis of schizophrenia proposes that a deficiency in glutamatergic neurotransmission and a resulting disturbance in balance between glutamatergic and dopaminergic systems within the basal ganglia may play a key role in the pathophysiology of schizophrenia (Kim et al 1980; Kornhuber et al 1989a). Zinc inhibits transmission at the NMDA receptor (Yeh et al 1990). Enhanced activity of zinc at the NMDA receptor might thus be involved in the pathophysiology of schizophrenia. Results of direct measurements of zinc in human postmortem brain tissue are inconsistent. Kimura and Kumura (1965) found a 50\% decrease in the zinc concentration in the hippocampus of schizophrenic patients and McLandy (1973) found a 30\% reduction in zinc content in the brains of patients with early onset schizophrenia. Greiner et al (1975) found no differences in zinc content in several brain regions, however, the hippocampus was not investigated by these authors. No consistent differences in the content of zinc were found in cerebrospinal fluid (Potkin et al 1982) or plasma (Gillin et al 1982) of schizophrenic patients.

There are several findings linking schizophrenia to disturbed iron metabolism. Iron exerts a profound influence on dopaminergic neurotransmission and behavior in laboratory animals (Yehuda and Youdim 1988). Low serum iron levels were reported in patients suffering from neurolepticinduced side effects, that is, akathisia (Brown et al 1987) and neuroleptic malignant syndrome (Rosebush and Mazurek 1991). Some investigators had the impression of increased iron staining in postmortem brain sections (Josephy 1930; Stevens 1982). Recently, iron was quantified in postmortem brains from schizophrenic patients using optical density measurements of coronal sections stained with the Pearl's technique (Casanova et al 1992). In that study staining intensity of iron was significantly increased in the caudate nucleus, which was attributed to neuroleptic therapy.

The main support for a copper hypothesis of schizophrenia comes from repeated findings of elevated copper and coeruloplasmin concentrations in the serum of schizophrenic patients (Bowman and Lewis 1982). Several copper-dependent enzymes are involved in catecholamine metabolism. More recent investigations, howcver, found unchanged copper levels in plasma and urine (Gillin et al 1982), cerebrospinal fluid (Shore et al 1983), and brain (Greiner et al 1975) of schizophrenic patients.

Besides the above-mentioned transition metals, changes in calcium and magnesium levels have also been linked to the pathophysiology of schizophrenia (Alexander and Jackson 1981). Direct measurements of these metals in postmortem human brain tissue have revealed no significant abnormalities in schizophrenia (Greiner et al 1975), however. The aim of the present study is to assess the content of iron, copper, zinc, magnesium, and calcium in several brain regions of schizophrenic patients and controls.

\section{Materials and Methods}

Postmortem handling of the autopsy material was similar in all cases. Brains were obtained at autopsy from 12 subjects ( 10 women, 2 men) with no history of neurological or psychiatric disorders. Control subjects had a mean age of $75.3 \pm 7.1$ years ( \pm SD, range 41-91 years). This group was compared to 11 schizophrenic patients (6 women, 5 men) diagnosed according to both Feighner et al (1972) and to the International Classification of Diseases (ICD-9). These patients had a mean age of $69.6 \pm 8.2$ years (range 57-80 years). The diagnostic subgroups according to the ICD-9 were schizophrenia simplex (ICD 295.0, $n=1$ ), hebephrenic subtype (ICD 295.1, $n=1$ ), paranoid subtype (ICD 295.3, $n=1$ ), chronic schizophrenia (ICD 295.6, $n=$ 7), and schizoaffective psychosis (ICD 295.7, $n=1$ ). Because there may be racial difference in the metabolism of certain metals (Potkin et al 1982), only brain specimens from Caucasian patients were investigated. Histopathological examination was performed on all brains to exclude other abnormalities such as tumor, infarction, anoxia, brain atrophy, and Alzheimer's disease. Postmortem delay time (i.e., time between death and freezing) was less than $24 \mathrm{hr}$ in all cases. A detailed examination of case notes was made to establish whether the patients had received neuroleptic medication during the period leading up to death. Three patients had been drug-free for at least 1 year and 7 patients were drug free for at least 3 months. Putamen samples from all the schizophrenic patients and three of the controls had previously been analyzed for [ $\left.{ }^{3} \mathrm{H}\right]$ spiperone binding (Kornhuber et al 1989b). Brain tissue was taken from the caudate nucleus, hippocampus, amygdala, cortex, corpus mamillare, gyrus cinguli, and hypothalamus from both hemispheres. It was not possible to collect samples of all seven regions from every brain (Table 1) The tissue was quickly frozen and stored at $-70^{\circ} \mathrm{C}$ until analysis

Iron, copper, zinc, magnesium, and calcium were determined by an atomic absorption procedure (Stevens 1970). Thawed tissue was freeze-dried at $-60^{\circ} \mathrm{C}$ and $10^{-2} \mathrm{~T}$ for 24 hr. Thereafter, the dry tissue was weighed and dissolved in acid-washed vials with $1 \mathrm{ml}$ of $65 \%$ nitric acid p.a. at $110^{\circ} \mathrm{C}$. The evaporated dry residue was taken up into $5 \mathrm{ml}$ of the diluent. Atomic absorption spectroscopy was performed using a Zeiss PMQ II photometer.

Results are expressed as micrograms per gram dry weight. Mean values are given \pm SD. Nonparametric statistics (Mann-Whitney $U$-test, Fisher's exact probability test, Spearman's rank correlation, Kruskal-Wallis one-way ANOVA) were used throughout using the two-tailed approach. $P$-values higher than 0.05 were regarded as not 
Table I. Case Data and Results of Metal Analysis in Postmontem Human Brains of Schizophrenic Patients (S) and Controls (C)

\begin{tabular}{|c|c|c|c|c|c|c|c|c|c|}
\hline & & & Gender & & Iron & Copper & Zinc & Magnesium & Calcium \\
\hline & & $n$ & $\mathbf{M} \mathbf{F}$ & Age (yrs) & & & eg/g dry weight & & \\
\hline \multirow[t]{2}{*}{ Caudate nucleus } & $\mathbf{C}$ & 9 & $1 / 8$ & $72.7 \pm 14.7$ & $450.9 \pm 167.6$ & $26.0 \pm 6.6$ & $67.2 \pm 17.5$ & $589.2 \pm 126.4$ & $265.3 \pm 234.2$ \\
\hline & $\mathbf{S}$ & 11 & $5 / 6$ & $69.6 \pm 8.2$ & $384.9 \pm 139.2$ & $24.9 \pm 5.8$ & $59.5 \pm 15.5$ & $584.6 \pm 83.1$ & $247.1 \pm 212.7$ \\
\hline \multirow[t]{2}{*}{ Hippocampus } & C & 8 & In & $76.6 \pm 9.3$ & $221.9 \pm 42.7$ & $18.0 \pm 3.4$ & $86.3 \pm 13.0$ & $556.2 \pm 172.1$ & $368.9 \pm 210.3$ \\
\hline & $\mathbf{S}$ & 9 & $4 / 5$ & $70.8 \pm 7.6$ & $207.5 \pm 27.3$ & $19.9 \pm 4.0$ & $83.8 \pm 10.2$ & $582.4 \pm 147.2$ & $267.0 \pm 110.8$ \\
\hline \multirow[t]{2}{*}{ Amygdala } & $\mathbf{C}$ & 9 & $1 / 8$ & $75.6 \pm 15.1$ & $221.7 \pm 57.8$ & $20.6 \pm 11.2$ & $73.2 \pm 15.5$ & $1370.5 \pm 2458$ & $259.5 \pm 164.6$ \\
\hline & $\mathbf{S}$ & 11 & $5 / 6$ & $69.6 \pm 8.2$ & $211.6 \pm 45.7$ & $18.8 \pm 4.2$ & $72.9 \pm 14.6$ & $541.0 \pm 174.5$ & $219.9 \pm 89.7$ \\
\hline \multirow[t]{2}{*}{ Contex } & C & 3 & $1 / 2$ & $75.0 \pm 10.0$ & $219.7 \pm 13.0$ & $23.0 \pm 5.3$ & $76.2 \pm 9.4$ & $734.7 \pm 51.5$ & $610.0 \pm 223.6$ \\
\hline & $\mathbf{S}$ & 7 & $3 / 4$ & $68.9 \pm 8.6$ & $195.9 \pm 24.2$ & $22.5 \pm 2.7$ & $73.5 \pm 9.4$ & $612.4 \pm 133.9$ & $492.4 \pm 285.1$ \\
\hline \multirow[t]{2}{*}{ Corpus mamillare } & $\mathbf{C}$ & 5 & $1 / 4$ & $77.0 \pm 10.6$ & $303.2 \pm 122.1$ & $19.0 \pm 2.1$ & $44.4 \pm 9.5$ & $483.0 \pm 176.3$ & $495.4 \pm 118.8$ \\
\hline & $\mathbf{S}$ & 5 & $2 / 3$ & $67.6 \pm 10.2$ & $251.6 \pm 54.4$ & $24.1 \pm 4.9$ & $59.9 \pm 14.3$ & $625.6 \pm 106.6$ & $450.6 \pm 171.2$ \\
\hline \multirow[t]{2}{*}{ Gyrus cinguli } & C & 5 & $1 / 4$ & $78.8 \pm 8.8$ & $166.4 \pm 48.5$ & $20.6 \pm 2.2$ & $58.9 \pm 22.0$ & $604.2 \pm 80.6$ & $337.6 \pm 93.4$ \\
\hline & $\mathbf{S}$ & 6 & $2 / 4$ & $68.5 \pm 9.4$ & $235.8 \pm 111.3$ & $21.4 \pm 2.9$ & $75.5 \pm 15.7$ & $664.7 \pm 43.8$ & $516.3 \pm 232.2$ \\
\hline \multirow[t]{2}{*}{ Hypothalamus } & $\mathbf{C}$ & 4 & $0 / 4$ & $78.5 \pm 9.3$ & $160.4 \pm 58.7$ & $23.2 \pm 7.0$ & $44.5 \pm 10.4$ & $625.8 \pm 285.4$ & $714.5 \pm 891.6$ \\
\hline & $\mathbf{S}$ & 5 & $1 / 4$ & $71.0 \pm 8.0$ & $290.2 \pm 170.5$ & $23.2 \pm 6.7$ & $60.3 \pm 16.2$ & $450.0 \pm 64.8$ & $1173.0 \pm 1766.5$ \\
\hline
\end{tabular}

Tissue samples from the different regions were not available from all brains.

Therefore, the case data are lisled for each region separately. Mean values are given \pm SD.

No differences in the content of any of the five metals between contruls and schizophrenic patients were observed (Mann-Whitney $U$-test, $p>0.05$ ).

Table 2. Effect f $\mathrm{Bran}$ Region on Metal Concentration (by Kruskal-Wallis One-Way ANOVA)

\begin{tabular}{lccccc}
\hline & Iron & Copper & Zine & Magnesium & Calcium \\
\hline Controls & r & NS & c & NS & NS \\
Schizophrenics & a & NS & b & NS & c \\
Total Group & c & - & c & NS & $c$ \\
\hline
\end{tabular}

NS $=$ not significant. $a_{p}<0.05 .{ }^{*} p<0.01 . c_{p}<0.001$.

significant. Because of the explorative nature of the study, no $\alpha$-corrections were applied.

\section{Results}

Data on case histories and results are given in Table 1. Age and gender were not significantly different between controls and schizophrenic patients. When the control and schizophrenic groups were compared, no differences in the content of any of the five metals investigated were observed (Table 1).

Looking at the total group (controls and schizophrenics combined), magnesium was distributed homogeneously in the seven brain regions investigated (Table 2), but there were significant regional differences for the other metals. For iron and copper, we found higher concentrations in the caudate nucleus than in the hippocampus and amygdala. Zinc concentrations were highest in the hippocampus, vith decreasing values in the amygdala and caudate nucleus (Table 1).

The effect of gender was investigated for each brain region using the total group (controls and schizophrenics) and the schizophrenic subgroup, respectively. The low number of men in the controls did not allow a meaningful comparison in this group. Except for zinc, none of investigated metals were significantly different in men compared to women, either in the total group or in schizophrenics (data not shown). In the total group there was a gender difference in zinc content only in the hippocampus (76.2 \pm 6.2 in men versus $88.6 \pm 10.6$ in women, $\mu \mathrm{g} / \mathrm{g}$ dry weight, $p<0.01$ ). In schizophrenic patients, there was a gender difference in the caudate nucleus $(47.6 \pm 12.3$ in men versus $65.9 \pm 13.1$ in women, $\mu \mathrm{g} / \mathrm{g}$ dry weight, $p<0.05$ ) and in the hippocampus $(77.1 \pm 6.8$ in men versus $88.3 \pm 8.8$ in women, $\mu \mathrm{g} / \mathrm{g}$ dry weight, $p<0.05$ ). There was no correlation between the neuroleptic-free period before death and the content of any of the metals investigaled, except ior a positive correlation between copper and the neurolepticfree period in the hippocampus $(r=0.83, p<0.02)$. One schizophrenic female aged 77 years had suffered from tardive dyskinesia before death. In this patient, the contents of all metals investigated were within the mean \pm 2 SD range of schizophrenic patients without tardive dyskinesia.

\section{Discussion}

The metal concentrations and the regional distributions found in this study were generally in accordance with previous investigations on human brain material (Harrison et al 1968; Völkl and Ule 1972; Ule et al 1974; Riederer et al 1989). In none of the brain regions investigated significant differences were observed between schizophrenics and controls. Because a number of the patients will have received long-term antipsychotic drug treatment, it is conceivable that this may have had an effect on the content of the metals investigated. Theoretically, a preexisting difference in the metal content could have been masked by neuroleptic drugs. Except for the copper content in the hippocampus, however, the neuroleptic-free time before death had no impact on the metal concentrations. The functional signifi- 
cance of the positive correlation between copper level and neuroleptic-free time before death should not be over-interpreted because of the explorative nature of the study (no $\alpha$-corrections were applied).

Taken together, the results of the present study show that

\section{References}

Alexander PE, Jackson AH (1981): Calcium and magnesium: relationship to schizophrenia and neuroleptic-induced extrapyramidal symptoms. In Alexander PE (ed) Electrolytes and Neuropsychiatric Disorders. Lancester: MTP Press, pp 247-275.

Andrews RCR (1990): Unification of the findings in schizophrenia by reference to the effects of gestational zinc deficiency. Med Hypotheses 31:141-153.

Bowman MB, Lewis MS (1982): The copper hypothesis of schizophrenia: A review. Neurosci Biobehav Rev 6:321-328.

Brown KW, Glen SE, White T (1987): Low serum iron status and akathisia. Lancet I:1234-1236.

Casanova MF, Comparini SO, Kim RW, Kleinman JE (1992): Staining intensity of brain iron in patients with schizophrenia: A postmortem study. J Neuropsychiatry Clin Neurosci 4:3641.

Constantinidis J (1991): The hypothesis of zinc deficiency in the pathogenesis of neurofibrillary tangles. Med Hypotheses 35:319-323.

Feighner JP, Robins E, Guze SB, Woodnuff RA, Winokur G, Munoz R (1972): Diagnostic criteria for use in psychiatric research. Arch Gen Psychiatry 26:57-63.

Gillin JC, Carpenter WT, Hambridge KM, Wyatt RJ, Henkin RI (1982): Zinc and copper in patients with schizophrenia. L'Encéphale 8:435-444.

Good PF, Perl DP, Bierer LM, Schmeidler J (1992): Selective accumulation of aluminum and iron in the neurofibrillary tangles of Alzheimer's disease: a laser microprobe (LAMMA) study. Ann Neurol 31:286-292.

Greiner AC. Chan SC, Nicolson GA (1975): Human brain contents of calcium, copper, magnesium and zinc in some neurological pathologies. Clin Chim Acta 64:211-213.

Harrison WW, Netsky MG, Brown MD (1968): Trace elements in human train: copper, zinc, iron, and magnesium. Clin Chim Acta 21:55-60.

Josephy H (1930): Dementia praecox (Schizophrenie). In Bumke $\mathbf{O}$ (ed) Die Anatomie der Psychosen. Berlin: Springer-Verlag.

Kim JS, Komhuber HH, Schmid-Burgk W, Holzmüller B (1980): Low cerebrospinal fluid glutamate in schizophrenic patients and a new hypothesis on schizophrenia. Neurosci Lett 20:379382.

Kimura K, Kumura J (1965): Preliminary report on metabolism of trace elements in neuropsychiatric diseases. 1. Zinc in schizophrenics. Proc Jap Acad Sci 41:943-949.

Koh J-Y, Choi DW (1988): Zinc alters excitatory amino acid neurotoxicity on cortical neurons. J Neurosci 8:2164-2171.

Kornhuber J, Mack-Burkhardt F, Riederer P, et al (1989a): $\left[{ }^{3} \mathrm{H}\right] \mathrm{MK}-801$ binding sites in postmortem brain regions of schizophrenic patients. J Neural Transm 77:231-236.

Kornhuber J, Riederer P, Reynolds GP, Beckmann H, Jellinger K. Gabriel E (1989b): 'H-Spiperone binding sites in post-mortem there are no profound differences in the content of iron, copper, zinc, magnesium, and calcium in brain tissue of controls and schizophrenic patients. The results are therefore in full agreement with the earlier study by Greiner et al (1975).

brains from schizophrenic patients: Relationship to neuroleptic drug treatment, abnormal movements, and positive symptoms. J Neural Transm 75:1-10.

McLandy T (1973): Hippocampal zinc in chronic alcoholism and schizophrenia. IRCS Med Sci 2:1010.

O'Halloran TV (1993): Transition metals in control of gene expression. Science 261:715-725.

Pfeiffer CC, lliev V (1972): Zinc deficiency and copper excess in the schizophrenias. Int Rev Neurobiol (Suppl)1:141-165.

Potkin SG, Shore D, Torrey EF, et al (1982): Cerebrospinal fluid zinc concentrations in ex-heroin addicts and patients with schizophrenia: some preliminary observations. Biel Psychiatry 17:1315-1322.

Riederer P, Sofic E, Rausch W-D, et al (1989): Transition metals, ferritin, glutathione, and ascorbic acid in Parkinsonian brains. $J$ Neurochem 52:515-520.

Rosebush PI, Mazurek MF (1991): Serum iron and neuroleptic malignant syndrome. Lancet 338:149-151.

Shore D, Potkin SG, Weinberger DR, et al (1983): CSF copper concentrations in chronic schizophrenia. Am J Psychiatry 140:754-757.

Smart TG, Constanti A (1990): Differential effect of zinc on the

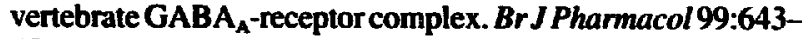
654.

Stevens BJ (1970): Clinical Applications of Atomic Absoption Spectroscopy. Australia: Varian Techtron.

Stevens JR (1982): Neuropathology of schizophrenia? Arch Gen Psychiatry 39:1 131-1139.

Ule G, Völkl A, Beriet H (1974): Spurenelemente im menschlichen Gehim. II. Kupfer-, Zink-, Calcium- und Magnesiumikonzentration in 13 verschiedener Hirnregionen während der 4. bis 8 . Lebenskdekade im Vergleich zum Hirneisen. Z Neurol 206:117-128.

Völkl A, Ule G (1972): Spurenelemente im menschlichen Gehim. Altersabhängigkeit der Eisenkonzentration in 13 verschiedenen Hirnregionen. ZNeurol 202:331-338.

Xie X,Smart TG (1991): A physiological role for endogenous zinc in rat hippocampal synaptic neurotransmission. Nature 349:521-524.

Yeh G-C, Bonhaus DW, McNamara JO (1990): Evidence that zinc inhibits $\mathrm{N}$-methyl-D-aspartate receptor-gated ion channel activation by noncompetitive antagonism of glycine binding. Mol Pharmacol 38:14-19.

Yehuda S, Youdim MBH (1988): Brain iron deficiency: biochemistry and behaviour. In Youdim MBH (ed) Brain Iron. Neurochemical and Behavioural Aspects. London: Taylor \& Francis, pp 89-114.

Yokoyama M, Koh J, Choi DW (1986): Brief exposure to zinc is toxic to cortical neurons. Neurosci Lett 71:351-355. 